\title{
Recent results from CMS
}

\section{Ezio Torassa*}

\section{on behalf of the CMS Collaboration}

INFN Sezione di Padova

Via F.Marzolo 8, 35131 Padova, Italy

E-mail: ezio.torassaepd.infn.itl

The CMS experiment has recently released results from a number of new physics searches and updated the invariant mass plots for the new boson discovered in July 2012, improving the signal significance. The data have been collected during the 2011 and 2012 runs of the Large Hadron Collider. The data samples used in the analysis corresponds to 5 inverse femtobarns for the protonproton collisions at $\sqrt{s}=7 \mathrm{TeV}$ and 12.2 inverse femtobarns for the collisions at $\sqrt{s}=8 \mathrm{TeV}$. The combination of the results has allowed CMS to increase the significance of the new boson signal up to 6.9 standard deviations. The updated measurement of the mass is $m_{H}=125.8 \pm 0.4$ (stat.) \pm 0.4 (sys) GeV. The parity measurement, performed with the study of the kinematic variables of the $\mathrm{ZZ}$ decay, is in favour of the positive parity hypothesis (Standard Model Higgs) with 2.5 standard deviations. Two new heavy flavour resonances have been observed. The search of the rare $B_{s} \rightarrow \mu^{+} \mu^{-}$and $B_{d} \rightarrow \mu^{+} \mu^{-}$decay channels achieved new limits close to the standard model branching ratios. The status of SUSY and Exotic physics searches are reported. New physics results with heavy ion beams are described.

International Winter Meeting on Nuclear Physics,

21-25 January 2013

Bormio, Italy

${ }^{*}$ Speaker. 


\section{Introduction}

The integrated luminosity collected by the CMS experiment with proton-proton collisions was $6.13 \mathrm{fb}^{-1}$ in 2011 with a center of mass energy of $7 \mathrm{TeV}$ and $23.3 \mathrm{fb}^{-1}$ in 2012 with a center of mass energy of $8 \mathrm{TeV}$. The recorded luminosities were respectively $5.55 \mathrm{fb}^{-1}$ and $21.8 \mathrm{fb}^{-1}$ corresponding to $93 \%$ of efficiency. At the end of 2011 the CMS experiment collected also some data with lead-lead collisions with a center of mass energy of $2.76 \mathrm{TeV}$ per nucleon, the recorded integrated luminosity was $157 \mu b^{-1}$. At the beginning of 2013 data with proton-lead collisions with a center of mass energy of $5.02 \mathrm{TeV}$ per nucleon have been collected, the recorded integrated luminosity was $31.13 n b^{-1}$. The integrated luminosity from 2010 up to the end of 2012 are shown in Fig. 1. The discovery of the new boson announced in July 2012 with invariant mass of about $125 \mathrm{GeV}$ was obtained using only $24 \%$ of the 2012 data, the updated results presented here used $56 \%$ of the 2012 data.
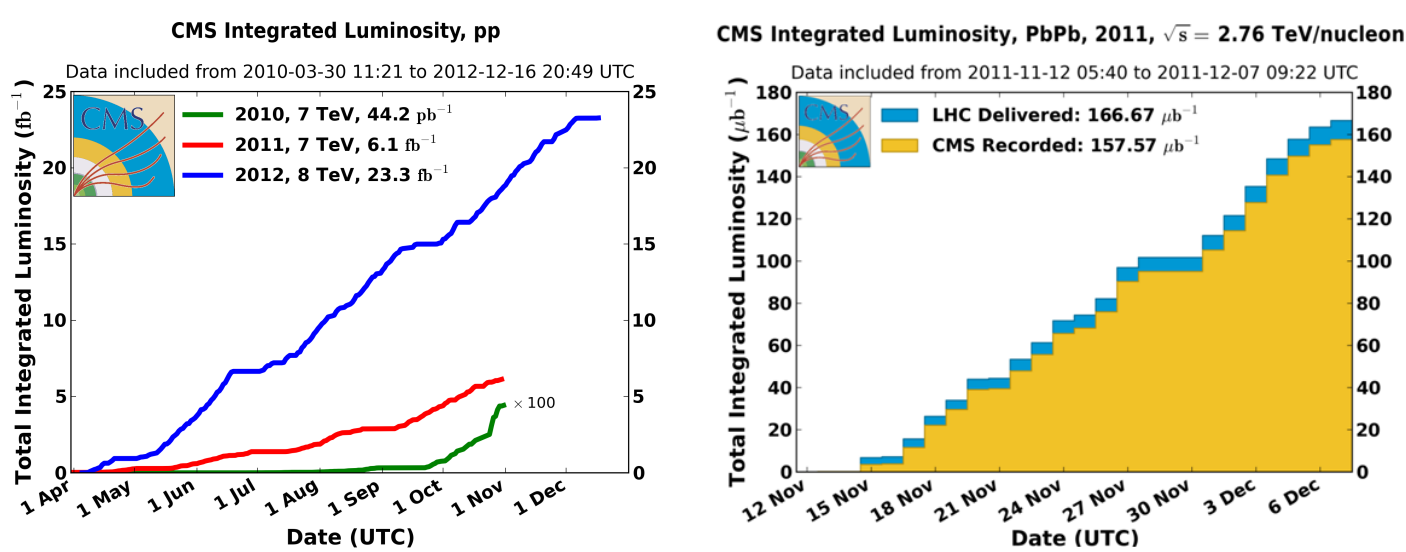

Figure 1: The CMS integrated luminosity from 2010 up to the end of 2012 for proton-proton (left) and lead-lead (right) collisions as a function of date of the year.

\section{The CMS Detector}

The Compact Muon Solenoid (CMS) is a multi-purpose detector designed to study protonproton collisions delivered by the CERN Large Hadron Collider. The detector is located in a underground cavern at a depth of $100 \mathrm{~m}$ at the site of Cessy, near the border of France and Switzerland. Particles emitted in hard collisions at the center of CMS cross in succession a silicon tracker, electromagnetic and hadron calorimeters, a solenoid magnet generating a megnetic field of $3.8 \mathrm{~T}$ and muon detectors embedded in the solenoid iron return yoke. Fig. 2 shows a sketch of the CMS experiment with all subdetectors. The momenta of charged particles are measured using a 13-layer silicon pixel and strip tracker which provides a track momentum resolution of $1 \%$ at $100 \mathrm{GeV}$. The electromagnetic calorimeter (ECAL) is composed of lead tungstate crystals $\left(\mathrm{PbWO}_{4}\right)$ and provides energy measurements of electromagnetic cascades with a resolution of about $3 \% / \sqrt{E}$. The hadron calorimeter (HCAL) is composed of brass-scintillator and provides energy measurements of hadronic cascades with a resolution of about $100 \% / \sqrt{E}$. These detectors consist of a barrel assembly covering the central region, plus two endcaps covering the solid angle for particles emitted 
at lower angle with respect to the beams direction. The outermost component of the CMS detector is the muon system, consisting of four layers of gas detectors placed within the steel return yoke. The muon system is composed by two detectors (Drift Tubes and Resistive Plate Chambers) in the barrel region, and two detectors (Cathode Strip Chambers and Resistive Plate Chambers) in the forward region. More detail on the CMS detector is provided elsewhere [1].

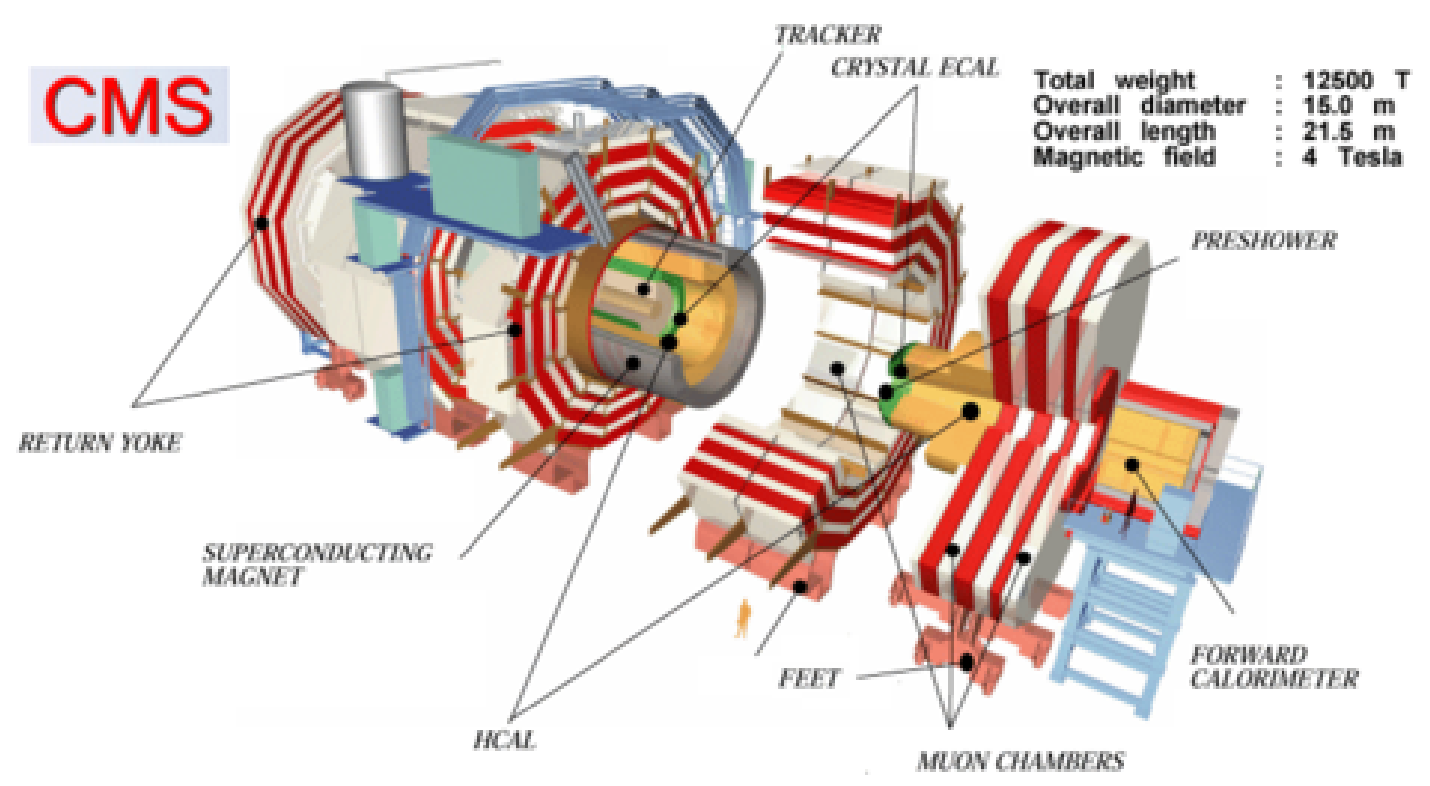

Figure 2: An exploded view of the CMS detector showing the outer muon chambers (grey) embedded in iron (red). Internally can be seen the hadronic calorimeter HCAL (orange) and the electromagnetic calorimeter ECAL (green). The tracker is located in the core of the central barrel.

\section{Measurements of Electroweak Observables}

The Electroweak physics signals provide experimental tool for the calibration of reconstracted physics objects. $\mathrm{W}$ and $\mathrm{Z}$ decays to leptons provide calibration of the reconstruction of electrons, muons, taus, and missing transverse energy $E_{T}^{m i s s}$ from neutrinos. Tag-and-probe techniques using $\mathrm{Z}$ decays allow to reduce systematic uncertainty on efficiency estimates. The number of produced $\mathrm{W}(\mathrm{Z})$ boson is about 100 millions (30 millions) every $\mathrm{fb}^{-1}$ of integrated luminosity. The production cross section of $\mathrm{W}$ and $\mathrm{Z}$ bosons at a centre-of-mass energy of $7 \mathrm{TeV}$ (and $8 \mathrm{TeV}$ ) has been studied both inclusively [2],[3] and as a function of the number of hadronic jets accompanying the bosons [4]. Additional measurements have determined the cross sections of $W \gamma$ and $Z \gamma$ production [5], as well as the production rates of $W W, W Z$, and $Z Z$ pairs [6],[7],[8],[9],[10],[11]. In all these measurements, $\mathrm{W}$ boson candidates are selected by searching for their decay to $e v$ and $\mu \nu$ final states, and $Z$ bosons by searching for their ee and $\mu \mu$ final states. In the case of $Z Z$ production, however, the second boson has also been identified in its decay to $\tau$ lepton pairs. The results are in excellent agreement with theoretical calculations, which are available at next-to-leading order (NLO) and next-to-next-to-leading order (NNLO) in perturbative QCD. Figure 3 provides a summary of the CMS measurements of these processes. 


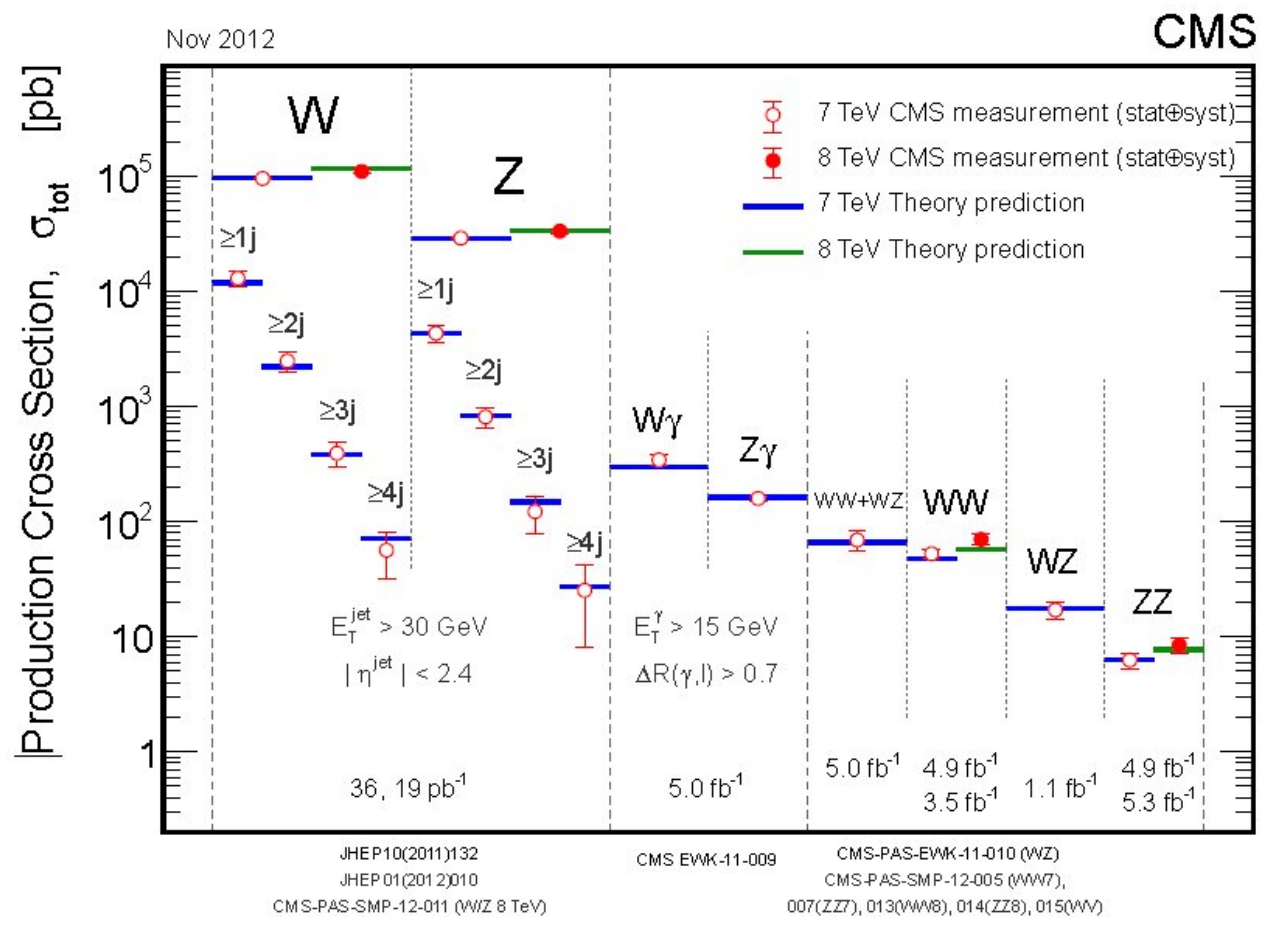

Figure 3: The total production cross section of several final with bosons in picobarns. The red empty markers are the $7 \mathrm{TeV}$ data, the red full markers are the $8 \mathrm{TeV}$ data. The measurements are compared with the theoretical predictions (blue lines for $7 \mathrm{TeV}$ and green lines for $8 \mathrm{TeV}$ ). For single boson production are also reported the cross sections of processes including one to at least four hadronic jets with transverse energy with $E_{T}>30 \mathrm{GeV}$.

\section{Searches for the Higgs Boson}

In July 2012 ATLAS [12] and CMS [13] announced the discovery of a new boson with a mass around $125 \mathrm{GeV}$. Five decay modes have been studied: $\gamma \gamma, Z Z, W W, \tau \tau$ and bb. The data sample used corresponds to about $5 \mathrm{fb}^{-1}$ at $7 \mathrm{TeV}$ and $5 \mathrm{fb}^{-1}$ at $8 \mathrm{TeV}$. The updated results for the last four channels with the increased data sample of $12 \mathrm{fb}^{-1}$ at $8 \mathrm{TeV}$ and the parity measurement using the ZZ decay channel are presented.

\subsection{Higgs production and decay}

The standard model Higgs boson has a non-zero coupling to all massive particles, and can therefore be produced by several different mechanisms in proton-proton collisions. From an experimental standpoint the most important reactions at the LHC are in the order of relevance: 1) gluon fusion diagrams, where a Higgs boson is emitted most frequently by a virtual top-quark loop; 2) vector-boson-fusion processes, where the Higgs is produced together with two characteristic highrapidity hadronic jets resulting from the emission of two virtual $\mathrm{W}$ or $\mathrm{Z}$ bosons off the initial state quarks; 3) Higgs-strahlung diagrams, where the particle is radiated by a highly-off-shell $\mathrm{W}$ or $\mathrm{Z}$ boson; 4) top antitop quarks associated production. A graph of the cross sections predicted for these processes as a function of the value of the Higgs boson mass is given in Fig. 4. 

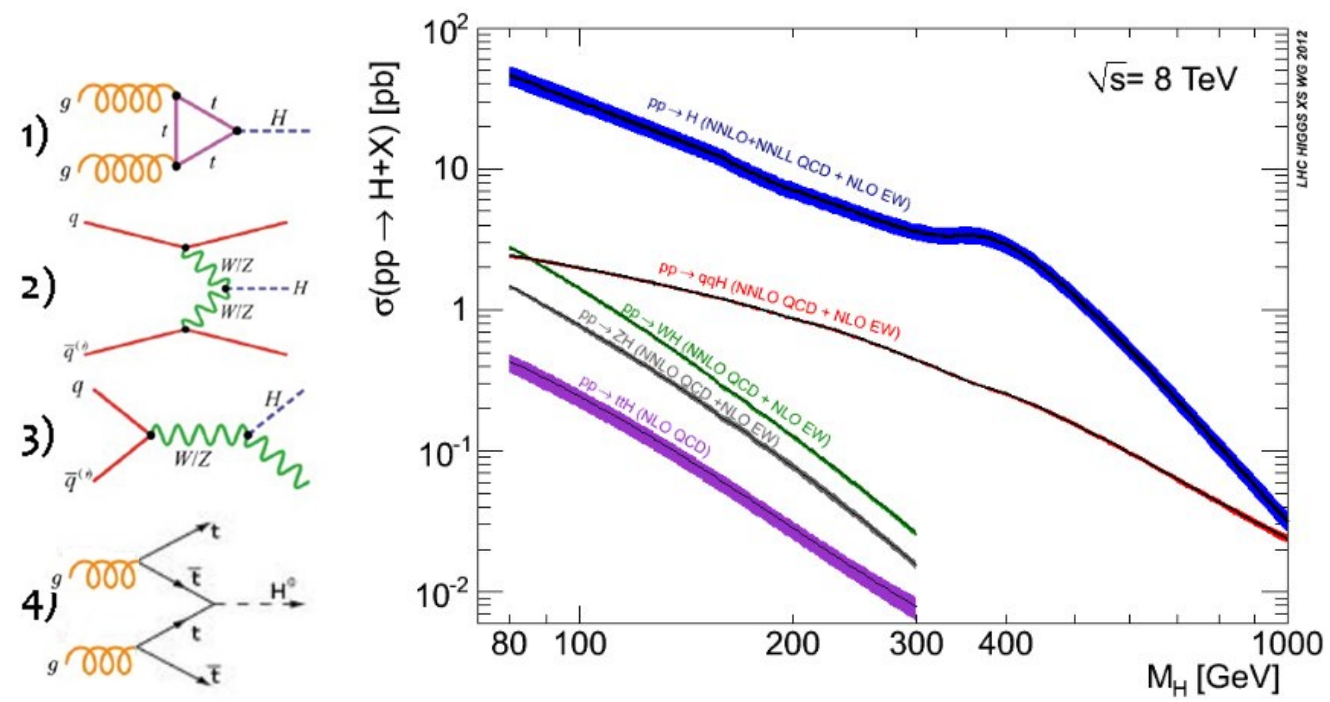

Figure 4: Predicted cross section of Higgs production processes in $8 \mathrm{TeV}$ proton-proton collisions as a function of Higgs boson mass. From top to bottom the curves describe gluon-fusion, vector-boson fusion, associated $\mathrm{WH}$ and $\mathrm{ZH}$ production and top antitop quarks associated production
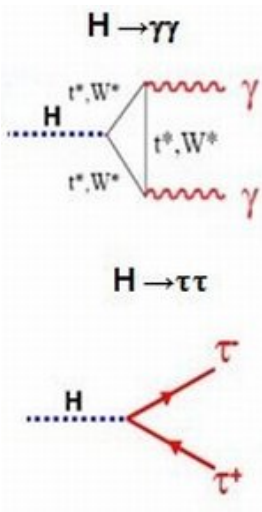
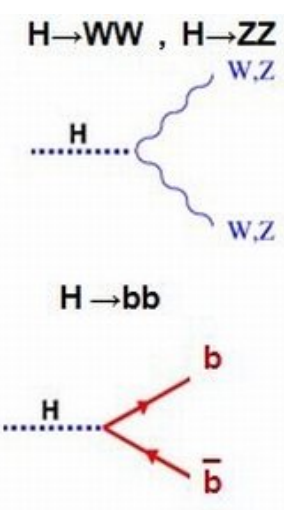

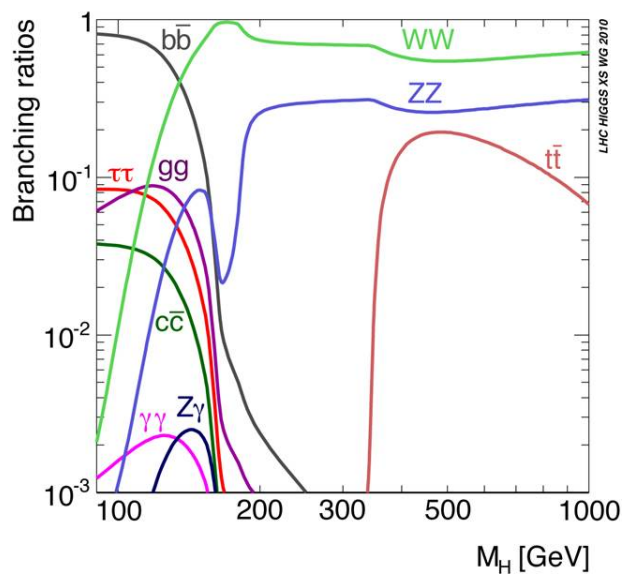

Figure 5: Higgs boson branching fractions as a function of Higgs boson mass.

\subsection{Searches for $H \rightarrow \gamma \gamma$}

The branching fraction of the $H \rightarrow \gamma \gamma$ channel is small (Fig. 5), about $0.2 \%$ in the $120-130 \mathrm{GeV}$ mass range, yet the efficient and clean identification of energetic photons and their precise energy measurement allow to discriminate the Higgs invariant mass peak over the large background contribution. The analysis is as published in Ref. [13] and has not been updated with additional data. The event sample is split into two mutually exclusive classes: diphoton events with one forward and one backward jet, consistent with the vector-boson fusion (VBF) topology, and all remaining events. This division is motivated by the consideration that there is a better signal-to-background ratio in the first class than in the second. The non-dijet class, containing over $99 \%$ of the data, is 
subdivided into four sub-classes based on the output of a multivariate discriminant that assigns a high score to signal-like events. Particular care is put in the determination of the hard interaction vertex. The average number of primary vertices due to interactions in 2011 (2012) collisions is about 10 (21). They are distributed along the beam direction within the standard deviation $\sigma_{Z} \sim 5-6$ $\mathrm{cm}$ of the LHC beam. The analysis requires that the vertex location is determined with a better than $1 \mathrm{~cm}$ precision in order to reduce to a negligible level the smearing of $m_{\gamma \gamma}$ resulting from uncertainty in the opening angle of the photon pair. The most probable vertex is identified with better than $80 \%$ efficiency using the kinematical properties of the observed charged tracks of each vertex as inputs to a Boosted Decision Tree (BDT) discriminant. The background in the signal region is estimated from a fit to the observed diphoton mass distribution in each of the five event classes. Figure 6 (left) shows the result of performing a weighted sum over all event classes, where the weights are the ratio of signal to background.

\subsection{Searches for $H \rightarrow Z Z$}

In the $H \rightarrow Z Z^{(*)} \rightarrow 4 \ell$ channel [14], we search for a four-lepton mass peak over a small continuum background. The results for this decay channel have been updated using $5.0 \mathrm{fb}^{-1}$ of pp data from the LHC collected in 2011 at $\sqrt{s}=7 \mathrm{TeV}$, and $12.2 \mathrm{fb}^{-1}$ collected in 2012 at $\sqrt{s}=8 \mathrm{TeV}$. The $4 e, 4 \mu$ and $2 e 2 \mu$ sub-channels are analysed separately since there are differences in the fourlepton mass resolutions and the background rates arising from jets misidentified as leptons. The dominant irreducible background in this channel is from non-resonant $\mathrm{ZZ}$ production. A kinematic discriminant was used to increase the separation between signal and background. The discriminant is constructed based on the probability ratio $\mathscr{P}_{\text {sig }} / \mathscr{P}_{\text {sig }}+\mathscr{P}_{b k g}$, the used variables are the masses of the dilepton pairs and the five angles fully defining a four-lepton configuration in their centerof-mass frame (Fig. 11). The distribution of the four-lepton reconstructed mass for the sum of the $4 e, 4 \mu$, and $2 e 2 \mu$ channels is shown in Fig. 6 (right).
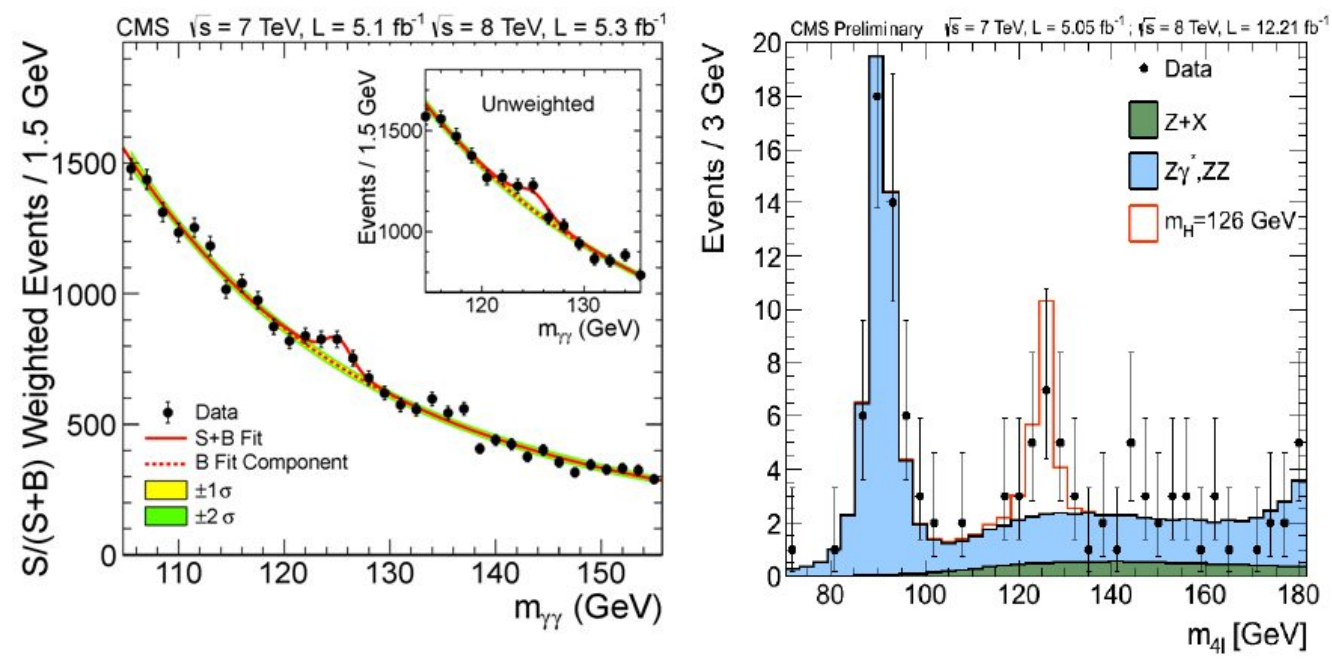

Figure 6: Invariant mass distributions for the $H \rightarrow \gamma \gamma$ (left) and for the $H \rightarrow Z Z$ (right) decay channels . 


\subsection{Searches for $H \rightarrow W W$}

The $H \rightarrow W W \rightarrow 2 \ell 2 v$ analysis [15], [16] searches for an excess of events with two leptons of opposite charge, large $E_{T}^{\text {miss }}$, and up to two jets. The results for this decay channel have been updated using 5.0 $\mathrm{fb}^{-1}$ of pp data from the LHC collected in 2011 at $\sqrt{s}=7 \mathrm{TeV}$, and $12.1 \mathrm{fb}^{-1}$ collected in 2012 at $\sqrt{s}=8 \mathrm{TeV}$. For events with no jets, the main background comes from nonresonant WW production; for events with one jet, the dominant backgrounds are from WW and topquark production, for the two-jet category the main background is from top-quark production. The events are split into same-flavour and different-flavour dilepton sub-channels, since the background from Drell-Yan production is much larger for the same-flavour dilepton events. The distributions of the transverse mass in the 0 -jet category adding the different-flavour final state is described in Fig. 7 for $\sqrt{s}=8 \mathrm{TeV}$. The transverse mass is the invariant mass between $W^{+} W^{-}$with the transverse missing energy used to estimate the neutrino momentum and all the $z$ components set to zero.

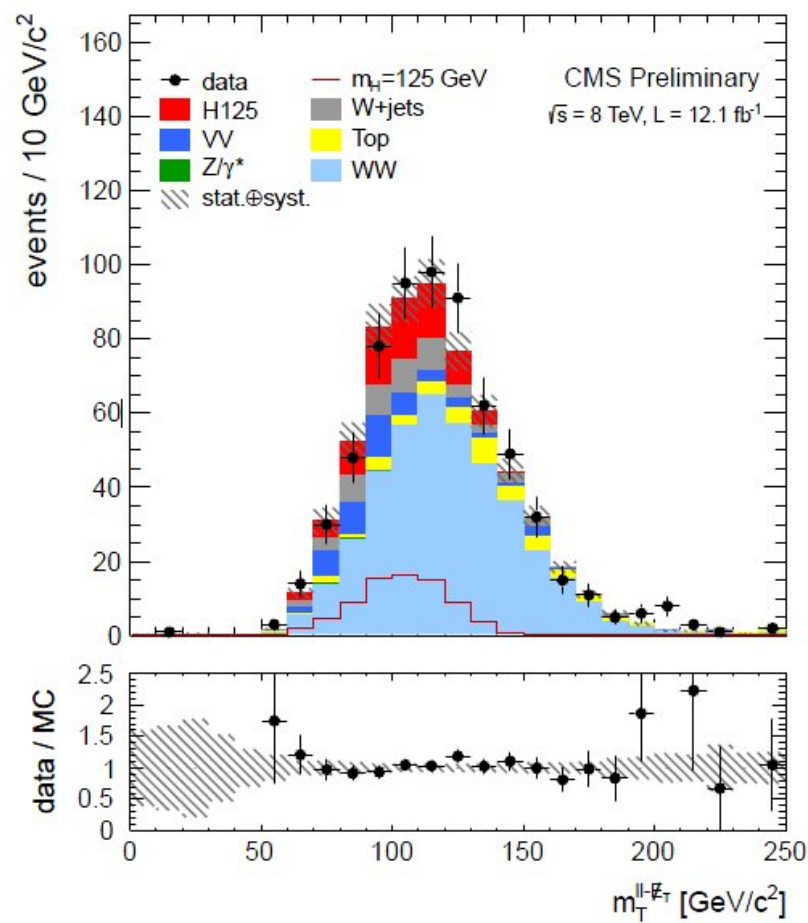

Figure 7: Distributions of the transverse mass in the 0-jet category adding the different-flavor final state for $\sqrt{s}=8 \mathrm{TeV}$; the expected signal for $m_{H}=125 \mathrm{GeV}$ and the main backgrounds are shown.

\subsection{Searches for $H \rightarrow b \bar{b}$ and $H \rightarrow \tau \tau$}

The $H \rightarrow b \bar{b}$ search [17] concentrates on Higgs boson production in association with a $\mathrm{W}$ or $\mathrm{Z}$ boson with the following decay modes: $W \rightarrow e^{ \pm} v / \mu^{ \pm} v$ and $Z \rightarrow e^{+} e^{-} / \mu^{+} \mu^{-} / \nu \bar{v}$. The $Z \rightarrow v \bar{v}$ decay is identified by requiring large $E_{T}^{\text {miss }}$. The Higgs boson candidate is reconstructed by requiring two b-tagged jets. The search for $H \rightarrow b \bar{b}$ is also performed using events where the Higgs boson is produced in association with a top-quark pair [18]. This analysis uses events where the top-quark pair decays to either the lepton-plus-jets $(t \bar{t} \rightarrow \ell v j j b b)$ or dilepton $(t \bar{t} \rightarrow v \ell v b b)$ 
final state. The $H \rightarrow \tau^{+} \tau^{-}$search [19] is performed using the final-state signatures $e \mu, \mu \mu, e \tau_{h}$, $\mu \tau_{h}, \tau_{h} \tau_{h}$, where electrons and muons arise from leptonic t-decays and $\tau_{h}$ denotes $\tau$ decaying hadronically.

\subsection{Combined results}

The statistical methodology used in this combination was developed by the ATLAS and CMS Collaborations in the context of the LHC Higgs Combination Group, the description of the methodology can be found in Refs. [20], [21]. The statistical hypothesys test to quantify the presence of an excess of events over what is expected for the background is described in 4.1:

$$
q_{0}=-2 \ln \frac{\mathscr{L}\left(n \mid b, \hat{\theta}_{0}\right)}{\mathscr{L}(n \mid \hat{\mu} \cdot s+b, \hat{\theta})}
$$

where $\mathrm{n}$ in the number of observed events, $\mathrm{s}$ stands for the signal expected under the SM Higgs hypothesis and $\mathrm{b}$ stands for backgrounds and $\theta$ are nuisance parameters describing systematic uncertainties. The signal $s$ is multiplied by a factor $\mu$, the value of the signal strength modifier which maximizes the maximum likelihood is one interesting indication of the compatibility of the observed events with the expected signal. The $\mathrm{p}$-value $\left(p_{0}\right)$ is the probability of an observed or more extreme results due to a statistical background fluctuation $p_{0}=P\left(q_{0} \geq q_{0}^{\text {obs }} \mid b\right)$. The p-values for the five decay mode and the overall combination are shown in Fig. 8 Table 1 summarises the median

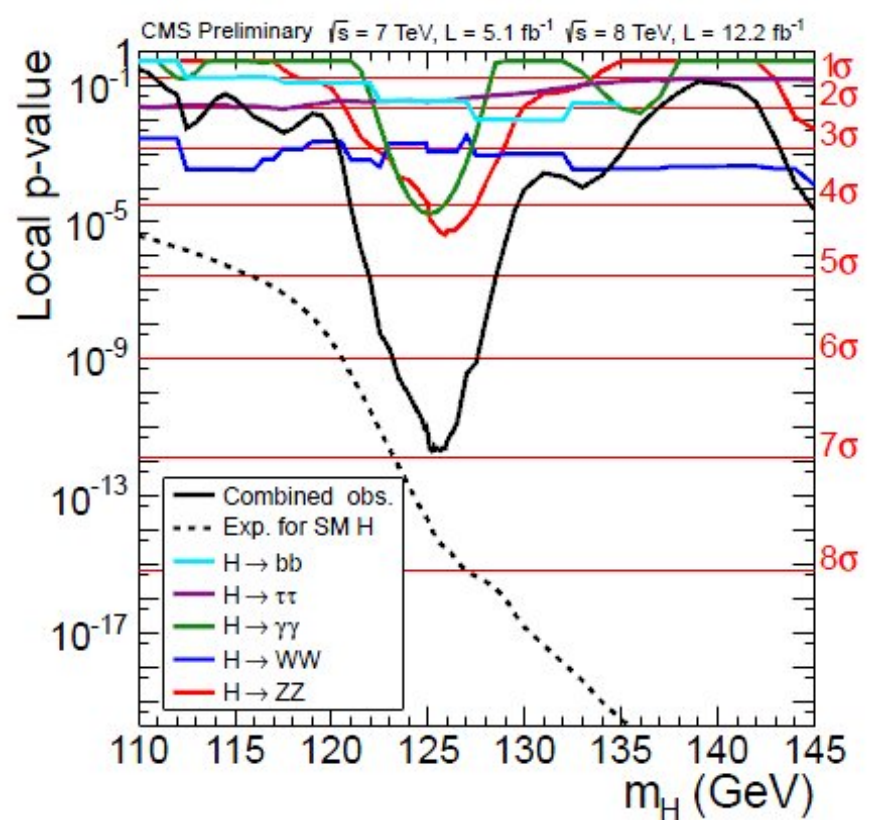

Figure 8: The observed local p-value for the five decay mode and the overall combination as a function of the SM Higgs boson mass in the range 110-145 GeV. The dashed line show the expected local p-values for a SM Higgs boson with a mass $m_{H}$.

expected and observed local significances for a SM Higgs boson mass hypothesis of $125.8 \mathrm{GeV}$ for the individual decay modes and their various combinations. The median expected significance is 


\begin{tabular}{l|c|c}
\hline Decay mode or combination & Expected $(\sigma)$ & Observed $(\sigma)$ \\
\hline$Z Z$ & 5.0 & 4.4 \\
$\gamma \gamma$ & 2.8 & 4.0 \\
$W W$ & 4.3 & 3.0 \\
$b b$ & 2,2 & 1.8 \\
$\tau \tau$ & 2.1 & 1.8 \\
\hline$\gamma \gamma+Z Z$ & 5.7 & 5.8 \\
\hline$\gamma \gamma+Z Z+W W+\tau \tau+b b$ & 7.8 & 6.9
\end{tabular}

Table 1: The significance of the median expected and observed event excesses in individual decay modes and their various combinations for a SM Higgs boson mass hypothesis of $125.8 \mathrm{GeV}$.

evaluated for a pseudo-observation equal to the median expected background plus signal rate. The significance for the 5 channel combination is $6.9 \sigma$. The mass of the observed state was measured using the $Z Z \rightarrow 4 \ell$ and $\gamma \gamma$ channels that have excellent mass resolution and for which the signal significances was $4.4 \sigma$ and $4.0 \sigma$, respectively. To extract the value of $m_{X}$ in a model-independent way, the signal strength modifiers for the $g g \rightarrow H \rightarrow \gamma \gamma, V B F+V H \rightarrow \gamma \gamma$, and $H \rightarrow Z Z \rightarrow 4 \ell$ processes are assumed to be independent and, thus, not tied to the SM expectation. Figure 9 shows the scan of the test statistic as a function of the hypothesised mass $m_{X}$ for the two final states separately and their combination. The combined mass measurement is $m_{X}=125.8 \pm 0.4$ (stat) \pm 0.4 (syst) GeV. Recently the CMS experiment updated the analysis using $5.0 \mathrm{fb}^{-1}$ collected in 2011 at

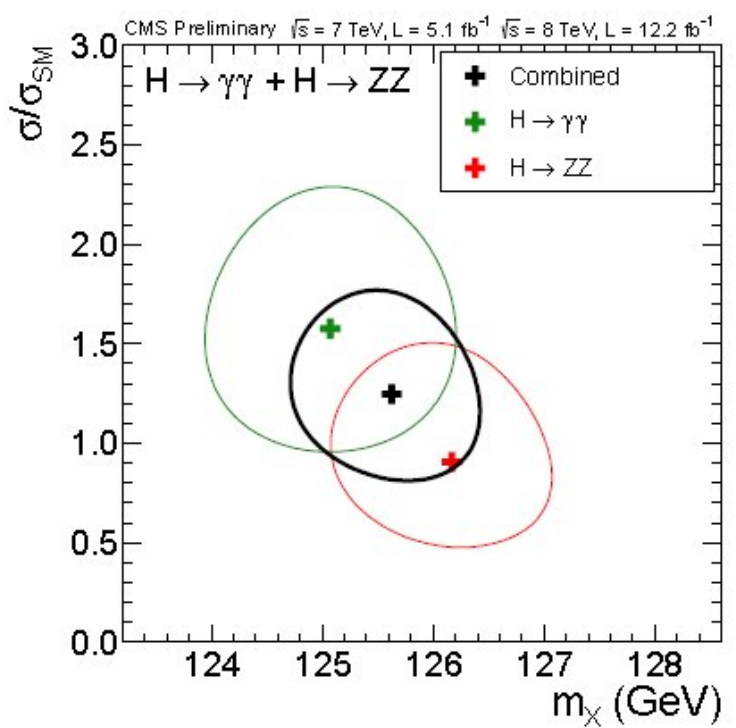

Figure 9: The $68 \%$ CL contours for the signal strength $\sigma / \sigma_{S M}$ versus the boson mass $m_{X}$ for the $\gamma \gamma$ and $4 \ell$ final states, and their combination.

$\sqrt{s}=7 \mathrm{TeV}$, and $19.6 f b^{-1}$ collected in 2012 at $\sqrt{s}=8 \mathrm{TeV}$ [22]. The updated mass measurement is $m_{X}=125.7 \pm 0.3$ (stat) \pm 0.3 (syst) GeV. The best fit values for the signal strength modifier $\mu=\sigma / \sigma_{S M}$ are shown in Fig. 10. The observed $\mu$ value for the combination for a hypothesised Higgs boson mass of $125.8 \mathrm{GeV}$ is found to be $0.88 \pm 0.21$ and is consistent with the value expected 

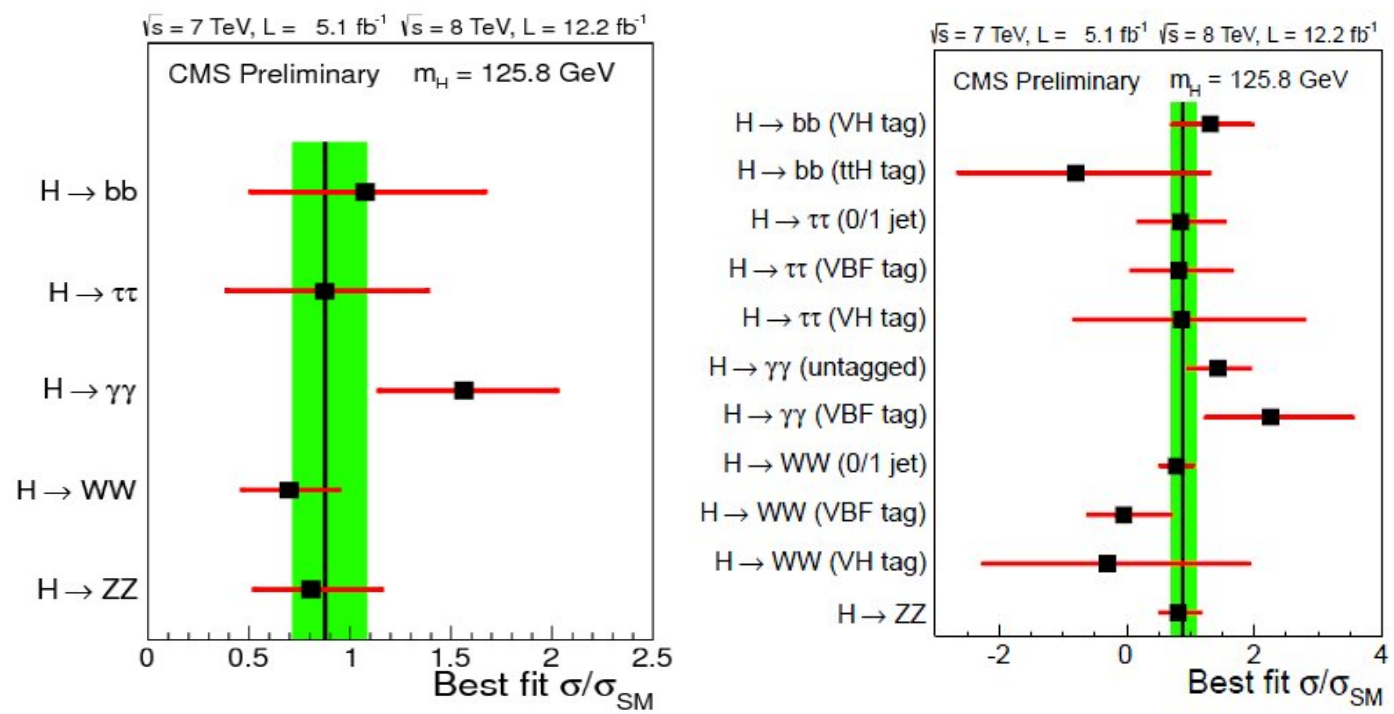

Figure 10: Values of $\sigma / \sigma_{S M}$ for the combination (solid vertical line), for individual decay modes (point) or sub-combinations of decay modes. The vertical band shows the overall $\sigma / \sigma_{S M}$ uncertainty.

for the SM Higgs boson $(\mu=1)$ within the $\pm 1 \sigma$ uncertainties. The updated measurement of the signal strength modifier in [22] is $0.80 \pm 0.14$.

\subsection{Higgs parity measurement}

For the decay channel $X \rightarrow Z Z \rightarrow 4 \ell$, the kinematic correlations between the four leptons are sensitive to the $J^{P}$ properties of the decaying resonance. The five considered angles $\vec{\Omega}\left(\theta^{*}, \Phi_{1}, \theta_{1}, \theta_{2}, \Phi\right)$ are described in Fig. 11. The state $J^{P}=0^{+}$is assumed to be a scalar with a coupling structure to

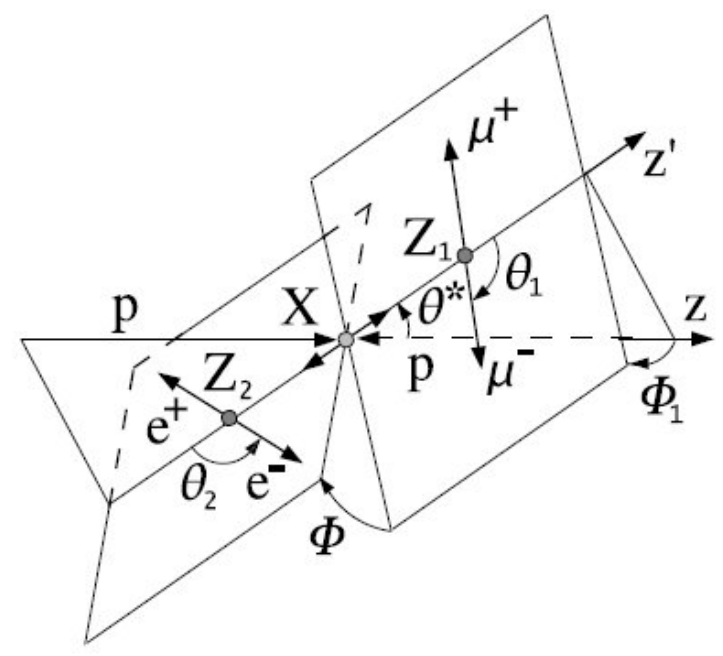

Figure 11: Description of the 5 angle in the $X \rightarrow Z Z \rightarrow 4 \ell$ decay used to discriminate between signal and background and between the scalar (SM Higgs) $J^{P}=0^{+}$and the pseudoscalar $J^{P}=0^{-}$hypothesis. 
gluons and $\mathrm{Z}$ bosons equal to that of the SM Higgs boson. The exact definitions of the coupling structure of the pseudo-scalar state $J^{P}=0^{-}$can be found in Ref. [14]. The discriminant $\mathscr{D}_{J^{P}}$ used to distinguish the SM Higgs $\left(J_{P}=0^{+}\right)$from one alternative hypothesis $J_{P}$ is the following:

$$
\mathscr{D}_{J^{P}}=\frac{P_{S M}}{P_{S M}+P_{J^{P}}}=\left[1+\frac{P_{J^{P}}\left(m_{1}, m_{2}, \vec{\Omega} \mid m_{4 \ell}\right)}{P_{S M}\left(m_{1}, m_{2}, \vec{\Omega} \mid m_{4 \ell}\right)}\right]
$$

Figure 12 (left) shows the $\mathscr{D}_{0^{-}}$("pseudo-MELA") distributions for the data, expected background and signal of two types, $0^{-}$and $0^{+}$, for $m_{H}=126 \mathrm{GeV}$. Figure 12 (right) shows the expected distributions of test statistic $q=-2 \ln \left(\mathscr{L}_{0^{-}+b k g} / \mathscr{L}_{0^{-}+b k g}\right)$ for background and the two signal hypothesis $\left(0^{-}\right.$and $\left.0^{+}\right)$. The data disfavour the pseudoscalar hypothesis $J^{P}=0^{-}$with 2.4 standard deviation $\left(C L_{s}=2.4 \%\right)$ and are consistent with with expectation, assuming $J^{P}=0^{+}$, within 0.5 standard deviations. The updated measurements [22] increased the distance to the $J^{P}=0^{-}$hypothesis up to 2.6 standard deviation and measured the distance to the $J^{P}=2^{+}$hypothesis to be 3.0 standard deviation.
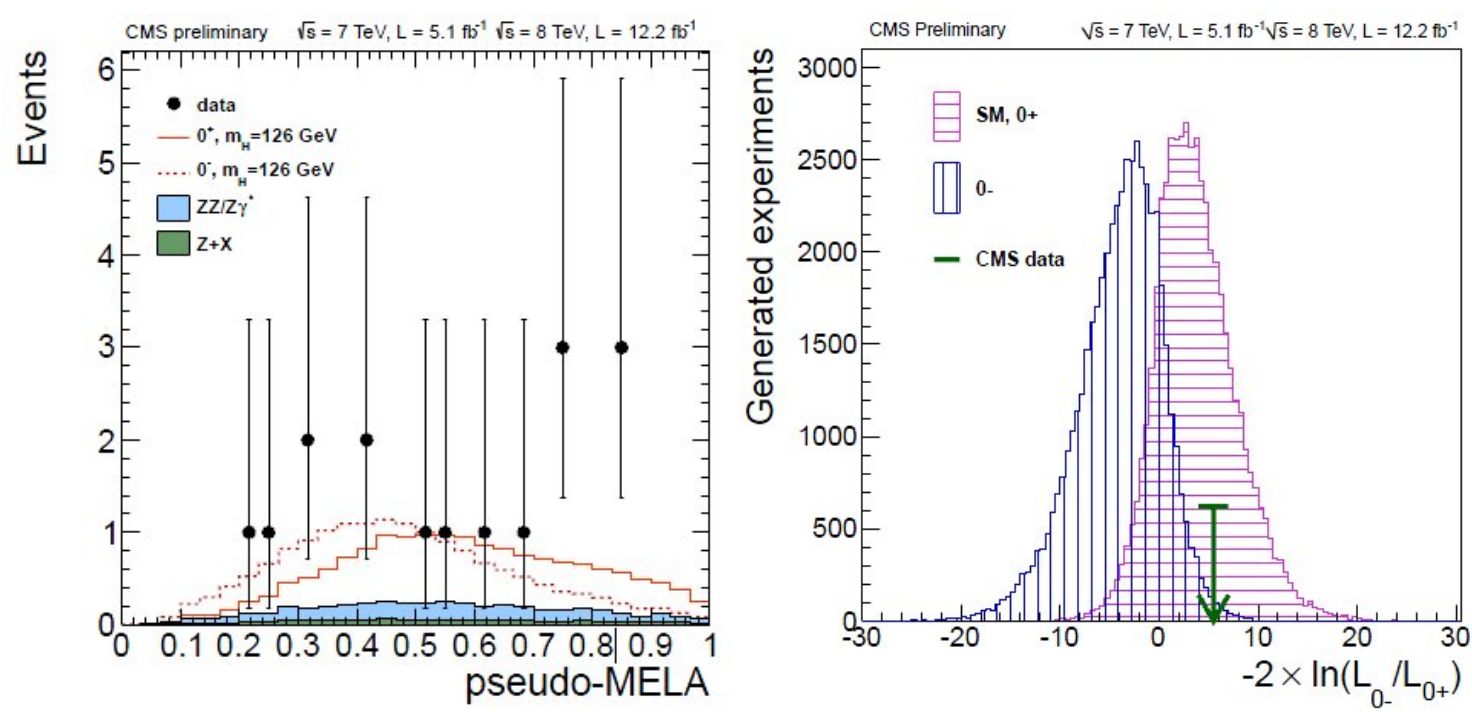

Figure 12: (Left) Distribution of $\mathscr{D}_{0^{-}}$("pseudo-MELA") for events in the mass range $106<m_{4 \ell}<141$ $\mathrm{GeV}$. (Right) Distribution of $q=-2 \ln \left(\mathscr{L}_{0^{-}} / \mathscr{L}_{0^{-}}\right)$for two signal types $\left(0^{+}\right.$horizontally hatched histogram, $0^{-}$vertically hatched histogram) for $m_{H}=126 \mathrm{GeV}$ shown with a large number of generated experiments. The arrow indicates the observed value.

\section{Results in Heavy Flavor Physics}

The discovery of new charmonium-like states over the last decade poses a serious challenge to the conventional quark model. Many explanations, such as charmed hybrids, tetraquark states, and molecular states, are proposed for these new entities. The CDF collaboration in 2009 reported evidence for a narrow structure, which they called $\mathrm{Y}(4140)$ in the exclusive decay $B^{+} \rightarrow J / \psi \phi K^{+}$ [23], [24]. A new search for states in the $J / \psi \phi$ mass spectrum from exclusive $B^{ \pm} \rightarrow J / \psi \phi K^{+}$ 
decays [25] is reported. The considered decays are $\phi \rightarrow K^{+} K^{-}$and $J / \psi \rightarrow \mu^{+} \mu^{-}$. Figure 13 shows the $\Delta M=\mathrm{m}\left(\mu^{+} \mu^{-} K^{+} K^{-}\right)-m\left(\mu^{+} \mu^{-}\right)$distributions. The two enhancements can not be due to reflections from other two body system decays. The mass and width are measured to be

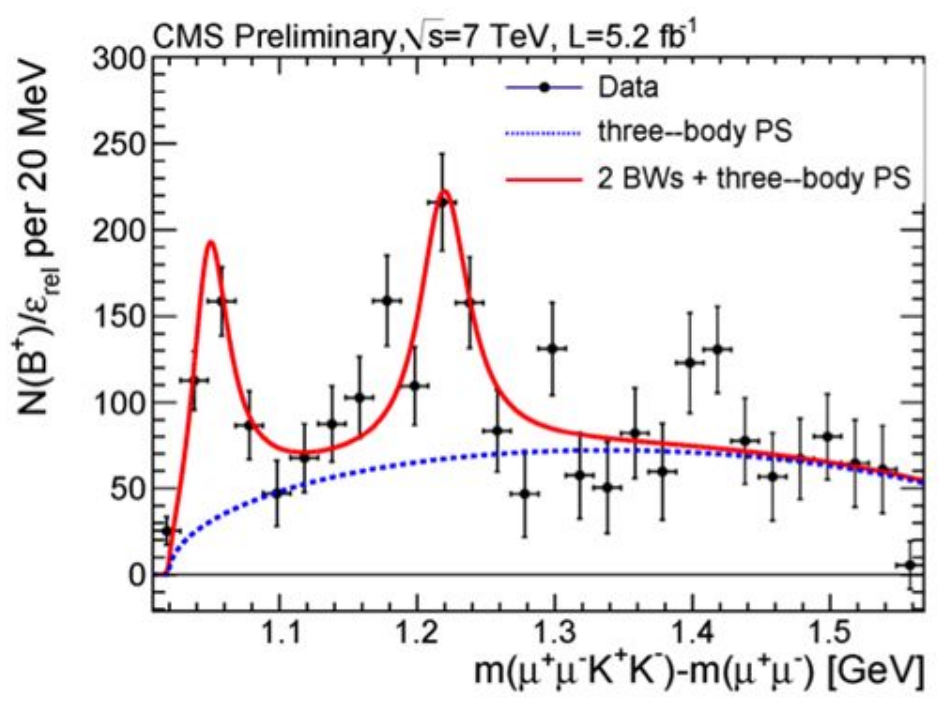

Figure 13: The mass difference $\Delta M$, between $\mu^{+} \mu^{-} K^{+} K^{-}$and $\mu^{+} \mu^{-}$, from the exclusive $B^{+} \rightarrow J / \psi \phi K^{+}$ signal.

$m_{1}=4148.1 \pm 2.7$ (stat) \pm 4.5 (syst) $\mathrm{MeV}$ and $\Gamma_{1}=30.0 \pm 11.7$ (stat) \pm 19.4 (syst) $\mathrm{MeV}$ for the first peak, $m_{1}=4316.9 \pm 4.1$ (stat) \pm 9.7 (syst) $\mathrm{MeV}$ and $\Gamma_{2}=34.6 \pm 16.7$ (stat) \pm 20.5 (syst) $\mathrm{MeV}$ for the second peak. The first resonance is compatible with the Y(4140) observed by the CDF experiment. The origin of these two resonances still must be understood: one "conventional" charmonium meson with these masses would be expected to decay predominantly into open charm pairs with a small branching fraction into $J / \psi \phi$, they can be more complex quantum status like $\mid D_{s}^{*+} D_{s}^{*-}>$ or exotic particles.

\subsection{Searches for $B_{s}^{0} \rightarrow \mu^{+} \mu^{-}$and $B_{d}^{0} \rightarrow \mu^{+} \mu^{-}$}

The rare decays $B_{s}^{0} \rightarrow \mu^{+} \mu^{-}$and $B_{d}^{0} \rightarrow \mu^{+} \mu^{-}$are highly suppressed in the standard model. Precise predictions of their branching fractions, $\mathscr{B}\left(B_{s}^{0} \rightarrow \mu^{+} \mu^{-}\right)=(3.23 \pm 0.27) \times 10^{-9}$ and $\mathscr{B}\left(B_{d}^{0} \rightarrow \mu^{+} \mu^{-}\right)=(1.07 \pm 0.10) \times 10^{-10}$ [26], make these modes powerful probes in the search for deviations from the SM. Several experiments already published upper limits for these two branching ratios (Fig. 14). The limits published by the CMS experiment are $\mathscr{B}\left(B_{s}^{0} \rightarrow \mu^{+} \mu^{-}\right)<7.7 \times 10^{-9}$ and $\mathscr{B}\left(B_{d}^{0} \rightarrow \mu^{+} \mu^{-}\right)<1.8 \times 10^{-9}$ at $95 \%$ CL [27]. They were obtained with $5.0 \mathrm{fb}^{-1}$ of data recorded in 2011 at $\sqrt{s}=7 \mathrm{TeV}$.

Recently LHCb published the first observation of the $B_{s}^{0} \rightarrow \mu^{+} \mu^{-}$decay channel [28]. The measured branching fraction $\left.\mathscr{B}^{L H C b}\left(B_{s}^{0} \rightarrow \mu^{+} \mu^{-}\right)=\left(3.2_{-1.2}^{+1.5}\right) \times 10^{-9}\right)$ is compatible with the standard model expectation. 


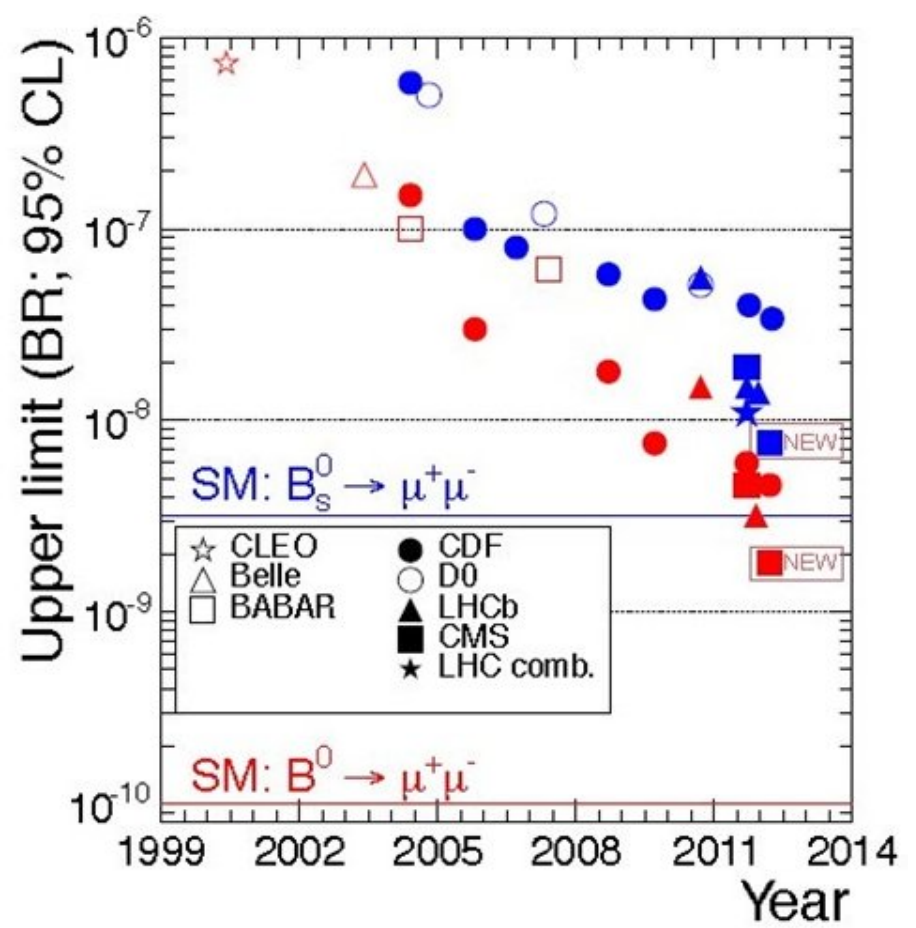

Figure 14: Upper limit branching ratios for the $B_{s}^{0} \rightarrow \mu^{+} \mu^{-}$and $B_{d}^{0} \rightarrow \mu^{+} \mu^{-}$decay channels measured by several experiments compared with the last CMS results.

\section{Searches for Supersymmetry signatures}

Among all hypothesized extensions of the standard model Supersymmetry is one of the most studied alternatives. The symmetry between standard model fermions and bosons with supersymmetric counterparts of bosonic and fermionic nature cancels the large quantum contributions to the Higgs boson mass due to virtual loops of standard model particles. The evidence of supersymmetric particles was still not found. Limits to the allowed mass of the hypothetical SUSY particles were published during recent years with higher and higher values. The CMS experiment has searched the 2011 data for supersymmetric particle signatures in different final states and with specific advanced methods [29],[31],[31]. In general SUSY particles can be produced in LHC proton-proton collisions in the form of pairs of squarks or gluinos like in Fig. 15, which carry colour quantum numbers and are thus subject to strong interactions. Depending on the mass spectrum of SUSY particles, the decay of squarks and gluinos may give rise to several lighter supersymmetric states in succession, with a typical "cascade" signature and characteristic kinematic features. Figure 16 summarizes the status of CMS searches for SUSY particles with $4.9 \mathrm{fb}^{-1}$ collected in 2011 at $\sqrt{s}=7 \mathrm{TeV}$. Upper limits in production cross sections are turned into exclusion regions in the plane described by the universal scalar and gaugino masses. The most sensitive searches are the one for jets and missing energy and the "razor" analysis, which exploits the kinematic configurations of the jets in the reconstructed reference frame of superparticle decay. New limits are now available using also the $8 \mathrm{TeV}$ data [32],[33],[34],[35]. 


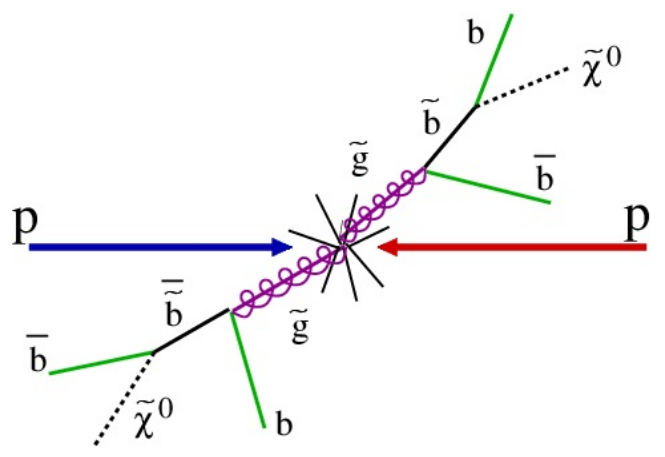

Figure 15: Example of one of the possible production (pairs of gluino) and decay chain (bottom-sbottom, bottom-neutralino) involving SUSY particles.

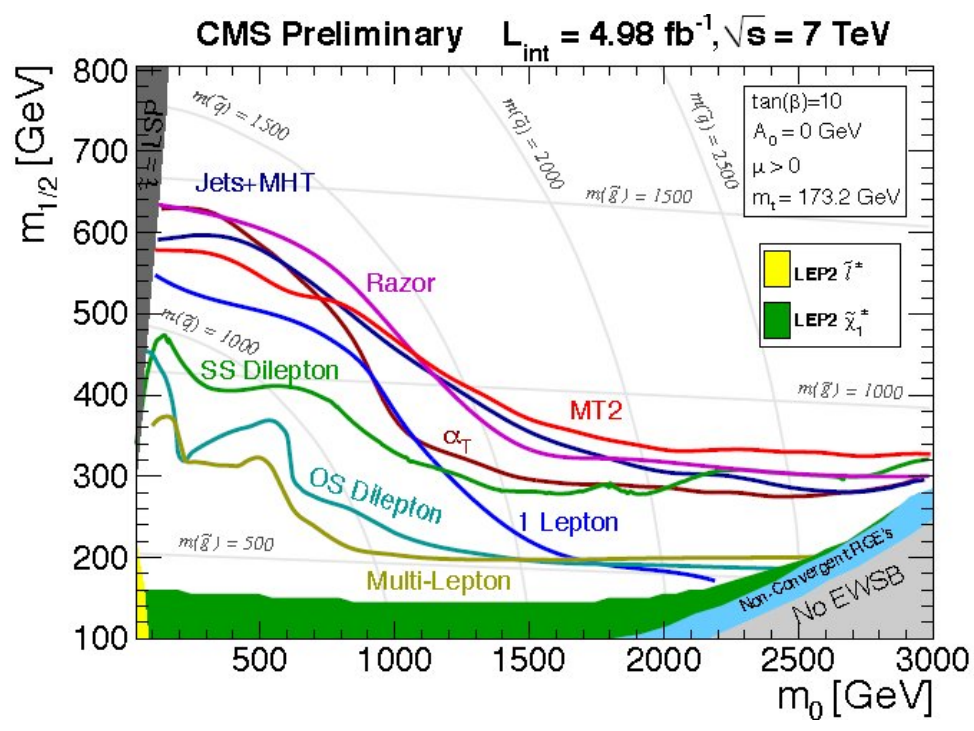

Figure 16: Summary of results of SUSY searches by CMS using 2011 data, here shown for a representative choice of SUSY parameters. Grey curves show iso-contours in the value of squark and gluino masses.

In addition to the direct searches strong constraints to the Supersymmentry come from the $B_{s} \rightarrow \mu \mu$ limits and from the Higgs boson mass measurements. One recent publication describes the exclusions due to these limits in the Constrained Minimal Supersymmetric Standard Model (CMSSM) parameter space [36]. In Fig. 17 gray points are the possible $\mathscr{B}\left(B_{s}^{0} \rightarrow \mu^{+} \mu^{-}\right)$values for a wide range of the CMSSM parameter space: $m_{0} ; m_{1 / 2} \in[50 ; 3000] \mathrm{GeV} ; \tan \beta \in[1 ; 60]$; $A_{0} \in[-10 ; 10] \mathrm{TeV} ; \operatorname{sign}(\mu)>0$. The green points are those in agreement with the Higgs mass constraint $\left(123 \mathrm{GeV}<M_{H}<129 \mathrm{GeV}\right)$ The horizontal line is LHCb measurement, the dotted line is $2 \sigma$ deviation. The $\mathscr{B}_{s}^{0} \rightarrow \mu^{+} \mu^{-}$admits a lower value of about $1.5 \times 10^{-9}$, which is still larger than the present experimental lower bound derived from the $\mathrm{LHCb}$ measurement however, once the Higgs mass limits are imposed, the vast majority of the allowed points are excluded. 

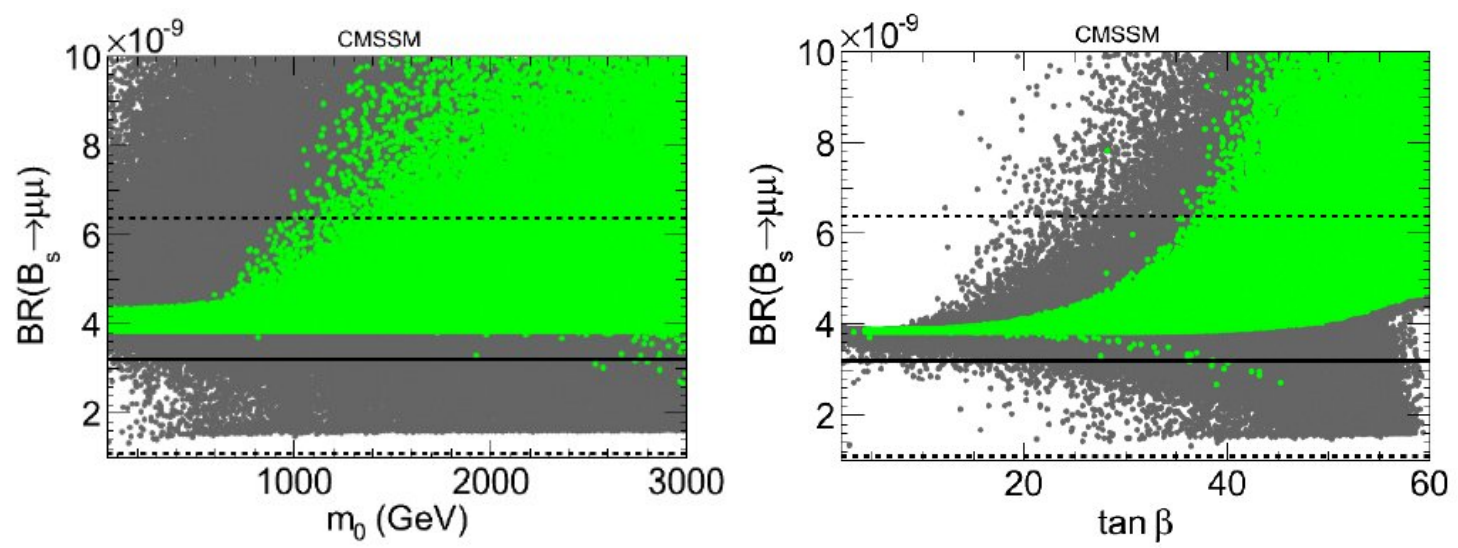

Figure 17: $\mathscr{B}\left(B_{s} \rightarrow \mu^{+} \mu^{-}\right)$vs. the CMSSM parameter $m_{0}$ (left) $\tan \beta$ (right)

\section{Exotic Searches}

A number of exotic extensions of the standard model have been tested with 2011 data: search for heavy bosons Z' and W' with different decay channels, search for heavy long-lived charged particles, search for Black Holes, search for heavy bottom-like quarks, search for quark compositeness (quarks composed by more fundamental entities), search for leptoquarks (scalar or vector bosons, which carry nonzero lepton and baryon numbers). In Fig. 18 as an example, the invariant mass distributions for $\mu^{+} \mu^{-}$and $e^{+} e^{-}$using data corresponding to about $4 \mathrm{fb}^{-1}$ collected at $\sqrt{s}=8 \mathrm{TeV}$ is shown. The resulting mass limit at $95 \%$ confidence level is $2590 \mathrm{GeV}$ for the $\mathrm{Z}$ ' in the Sequential Standard Model (SSM) hypothesis. The updated result using the full 2012 data set extended this limit up to $2960 \mathrm{GeV}$ [37].
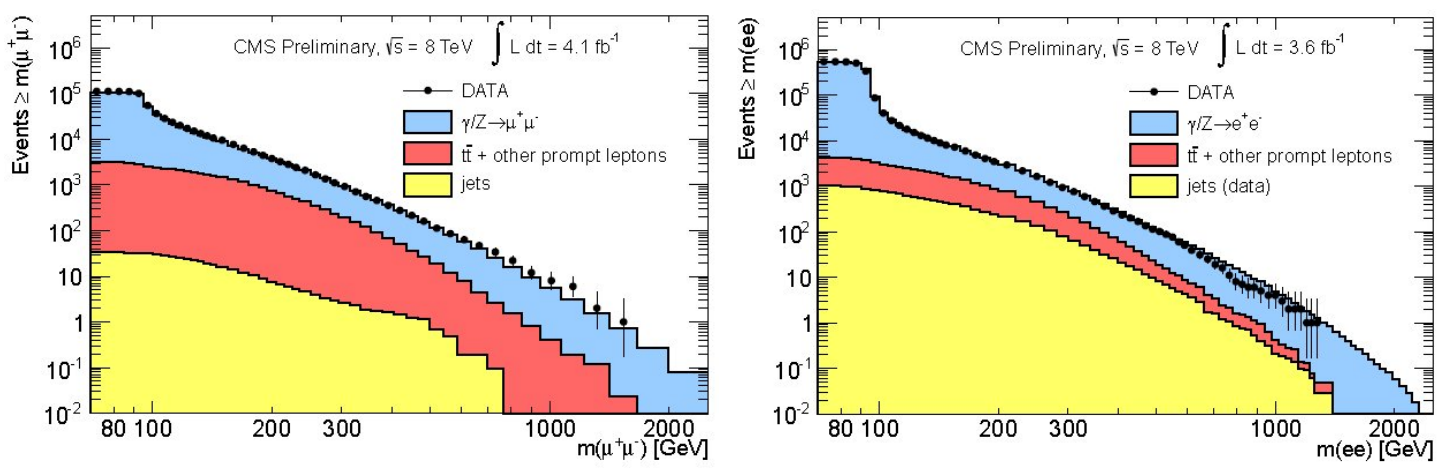

Figure 18: Invariant mass distributions for $\mu^{+} \mu^{-}$(left) and $e^{+} e^{-}$(right) with about $4 f b^{-1}$ collected data $\sqrt{s}=8 \mathrm{TeV}$ data.

In Fig. 19 only a summary of the limits from the recent searches using $5 \mathrm{fb}^{-1}$ at $\sqrt{s}=7 \mathrm{TeV}$ are provided. More stringed limits will be published with the $\sqrt{s}=8 \mathrm{TeV}$ data. The interested reader can refer to the public pages of the CMS experiment [38] for a more comprehensive list of results. 


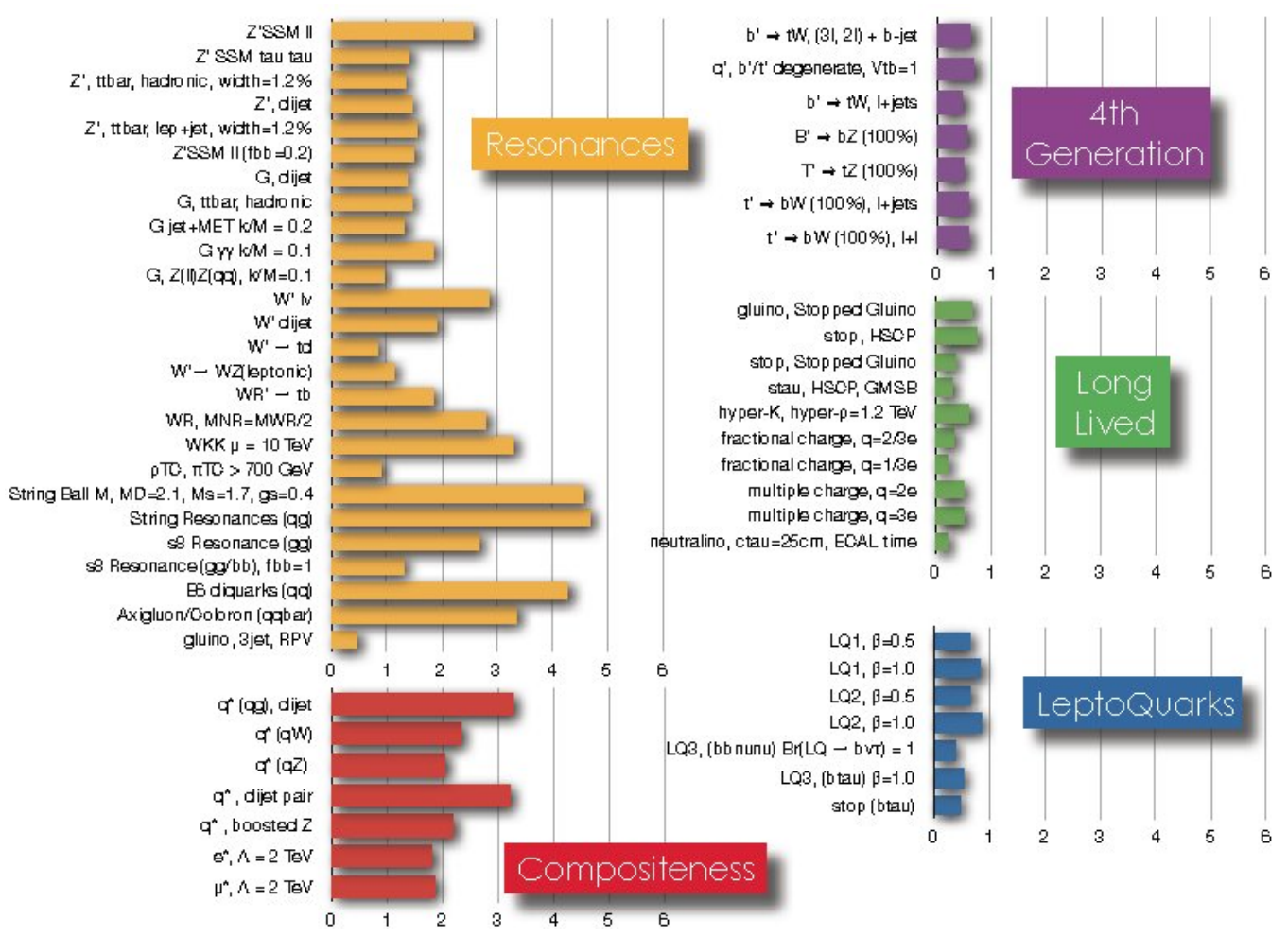

Figure 19: Summary of mass limits (in $\mathrm{TeV}$ ) for most of the CMS exotic searches with the collected data at $\sqrt{s}=7 \mathrm{TeV}$ data.

\section{Physics results with hevy ion beams}

Suppression of heavy quarkonium states has been proposed as a probe of the properties of the hot and dense medium created in high-energy heavy-ion collisions [39]. If a deconfined state, often referred to as the quark-gluon plasma (QGP), is formed, the confining potential of heavy quark-antiquark pairs is expected to be screened because of interactions with quarks and gluons in the medium. The resulting dissociation of the quarkonium states depends on the temperature of the medium, and is expected to occur sequentially, reflecting the increasing values of their binding energies. The $\mathrm{Y}(1 \mathrm{~S})$ is the most tightly bound quarkonium state, and is hence expected to be the one with the highest dissociation temperature. The suppression pattern was studied with the charmonium, the bottomonium family provides additional theoretical informations. Measurements of the absolute $\mathrm{Y}(\mathrm{nS})$ suppression and of the relative suppression of $Y(2 S)$ and $Y(3 S)$ with respect to $Y(1 S)$ has been published by $\mathrm{CMS}$ using $\mathrm{PbPb}(\mathrm{pp})$ data corresponding to an integrated luminosity of $150 \mu b^{-1}\left(230 n b^{-1}\right)$ collected in 2010 (2011) at the same center-of-mass energy per nucleon pair of $\sqrt{s_{N N}}=2.76 \mathrm{TeV}$ [40]. The dimuon invariant mass distributions of $\mathrm{PbPb}$ and pp interactions are shown in Fig. 20. The measurement of the ratio of the $Y(n S) / Y(1 S)$ ratios in $\mathrm{PbPb}$ and pp collisions benefits from an almost complete cancellation of possible acceptance or efficiency 
differences among the reconstructed resonances:

$$
\begin{aligned}
& \frac{Y\left(2 S /\left.Y(1 S)\right|_{P b P b}\right.}{Y\left(2 S /\left.Y(1 S)\right|_{p p}\right.}=0.21 \pm 0.07 \text { (stat) } \pm 0.02 \text { (syst) } \\
& \frac{Y\left(2 S /\left.Y(1 S)\right|_{P b P b}\right.}{Y\left(3 S /\left.Y(1 S)\right|_{p p}\right.}=0.06 \pm 0.06 \text { (stat) } \pm 0.06 \text { (syst) }
\end{aligned}
$$

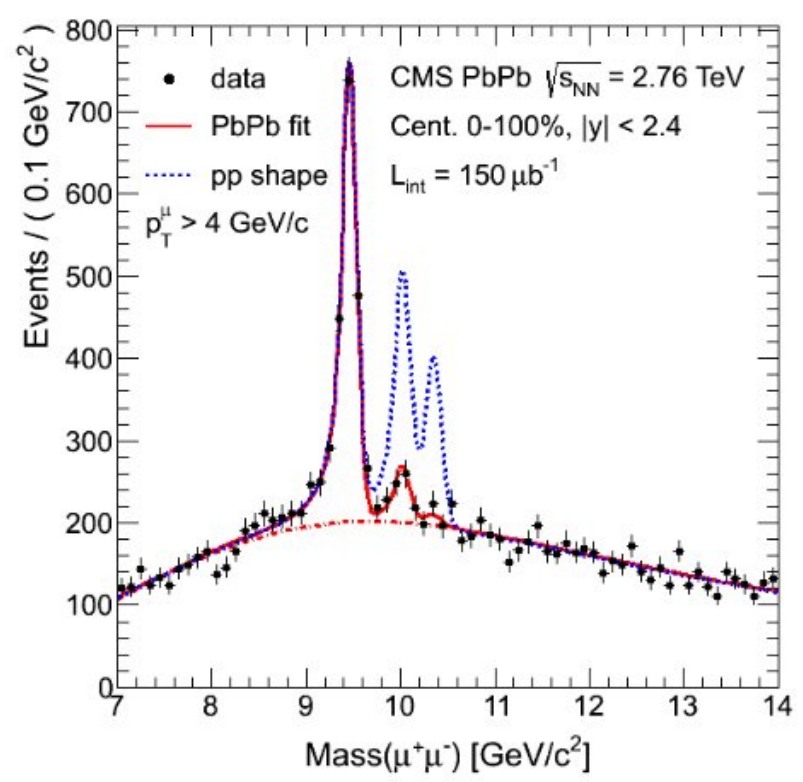

Figure 20: Dimuon invariant-mass distributions in $\mathrm{PbPb}$ data at $\sqrt{s_{N N}}=2.76 \mathrm{TeV}$. The blue dotted line is the shape obtained with pp interactions with the same energy. The same reconstruction algorithm and analysis selection are applied to both data sets.

\section{Conclusions}

The CMS results with 17.2 inverse femtobarns of collisions collected in the 2011 and 2012 proton-proton runs of the Large Hadron Collider have been described. Some updated results with about 25 inverse femtobarns have already been published, someone else will be published next months. The significance of the Higgs signal reached $6.9 \sigma$ and is equal or larger than $3 \sigma$ in three decay channels $(Z Z, \gamma \gamma$ and $W W)$. The mass of the Higgs boson is confirmed to be about $126 \mathrm{GeV}$ with one error of about $0.5 \mathrm{GeV}$. The parity measurement is in favour of the positive parity (SM) hypothesis with 2.5 standard deviations. Two new resonances were found, the first $Y(4140)$ confirms the CDF observation, the second $Y(4317)$ is a new observed resonance. New limits have been published for the $B_{d / s} \rightarrow \mu^{+} \mu^{-}$search, we expect to improve these measurements with the $\sqrt{s}=8 \mathrm{TeV}$ data to cross check to the LHCb observation. New limits for SUSY and Exotic searches have been published, providing at the moment no evidence for new physics. Suppression of $Y(n S)$ resonances in $\mathrm{PbPb}$ collisions have been confirmed and measured. 


\section{Acknowledgements}

We wish to congratulate our colleagues in the CERN accelerator departments for the excellent performance of the LHC machine. We thank the technical and administrative staff at CERN and other CMS institutes, and acknowledge support from: BMWF and FWF (Austria); FNRS and FWO (Belgium); CNPq, CAPES, FAPERJ, and FAPESP (Brazil); MES (Bulgaria); CERN; CAS, MoST, and NSFC (China); COLCIENCIAS (Colombia); MSES (Croatia); RPF (Cyprus); MEYS (Czech Republic); MoER, SF0690030s09 and ERDF (Estonia); Academy of Finland, MEC, and HIP (Finland); CEA and CNRS/IN2P3 (France); BMBF, DFG and HGF (Germany); GSRT (Greece); OTKA and NKTH (Hungary); DAE and DST (India); IPM (Iran); SFI (Ireland); INFN (Italy); NRF and WCU (Korea); LAS (Lithuania); CINVESTAV, CONACYT, SEP, and UASLPFAI (Mexico); MSI (New Zealand); PAEC (Pakistan); MSHE and NSC (Poland); FCT (Portugal); JINR (Armenia, Belarus, Georgia, Ukraine, Uzbekistan); MON, RosAtom, RAS and RFBR (Russia); MSTD (Serbia); SEIDI and CPAN (Spain); Swiss Funding Agencies (Switzerland); NSC (Taipei); TUBITAK and TAEK (Turkey); NASU (Ukraine); STFC (United Kingdom); DOE and NSF (USA).

\section{References}

[1] CMS Collaboration, The CMS experiment at the CERN LHC, JINST 3 (2008) S08004.

[2] CMS Collaboration, Measurements of the Inclusive $W$ and Z Production Cross Sections in pp Collisions at $\sqrt{s}=7 \mathrm{TeV}$ with the CMS experiment, J. High Energy Phys. 10 (2011) 132, arXiv:1107.4789.

[3] CMS Collaboration, Inclusive W/Z cross section at 8 TeV, CMS-PAS-SMP-12-011 (2012).

[4] CMS Collaboration, Jet Production Rates in Association with W and Z Bosons in pp Collisions at $\sqrt{s}=7$ TeV, J. High Energy Phys. 01 (2012) 010, arXiv:1110.4973.

[5] CMS Collaboration, Measurement of $W \gamma$ and $Z \gamma$ production in pp collisions at $\sqrt{s}=7$ TeV, Phys. Lett. B 701 (2011) 535-555, arXiv:1105.2758.

[6] CMS Collaboration, Measurement of the WW, WZ and ZZ cross sections at CMS, CMS-PAS-EWK-11-010 (2011).

[7] CMS Collaboration, Measurement of WW production rate, CMS-PAS-SMP-12-005 (2012).

[8] CMS Collaboration, Measurement of the ZZ production cross section and search for anomalous couplings in $2 \ell 2 \ell^{\prime}$ final states in pp collisions at $\sqrt{s}=7$ TeV, J. High Energy Phys. 01 (2013) 063 doi:10.1007/JHEP01(2013)063, arXiv:1211.4890.

[9] CMS Collaboration, Measurement of WW cross section at 8 TeV, CMS-PAS-SMP-12-013 (2012).

[10] CMS Collaboration, Measurement of ZZ cross section at 8 TeV, CMS-PAS-SMP-12-014 (2012).

[11] CMS Collaboration, $W W+W Z$ cross section in the $W+$ dijet channel, CMS-PAS-SMP-12-015 (2012).

[12] ATLAS Collaboration, Observation of a new particle in the search for the Standard Model Higgs boson with the ATLAS detector at the LHC, Phys. Lett. B 716 (2012) 1-29, doi:10.1016/j.physletb.2012.08.020, arXiv:1207.7214. 
[13] CMS Collaboration Collaboration, Observation of a new boson at a mass of $125 \mathrm{GeV}$ with the CMS experiment at the LHC, Phys. Lett. B 716 (2012) 30-61, doi:10.1016/j.physletb.2012.08.021, arXiv:1207.7235.

[14] CMS Collaboration, Discovery of a new boson in the search for the standard model Higgs bosons in the $H \rightarrow Z Z \rightarrow 4 \ell$ channel in pp collisions at $\sqrt{s}=7$ and $8 \mathrm{TeV}$, CMS Physics Analysis Summary CMS-PAS-HIG-12-041 (2012), arXiv:1212.6639.

[15] CMS Collaboration, Search for the Higgs Boson Decaying to $W^{+} W^{-}$in the Fully Leptonic Final State, Phys. Lett. B 710 (2012) 91-113, 10.1016/j.physletb.2012.02.076, arXiv:1202.1489.

[16] CMS Collaboration, Evidence for a particle decaying into $W^{+} W^{-}$in the fully leptonic final state in a standard model Higgs bosons search in pp collisions at $\sqrt{s}=8 \mathrm{TeV}$, CMS Physics Analysis Summary CMS-PAS-HIG-12-042 (2012).

[17] CMS Collaboration, Search for standard model Higgs bosons produced in association withWor Z bosons, and decayng to bottom quarks, CMS Physics Analysis Summary CMS-PAS-HIG-12-044 (2012).

[18] CMS Collaboration, Search for Higgs production in association with top quark pairs in pp collisions, CMS Physics Analysis Summary CMS-PAS-HIG-12-025 (2012).

[19] CMS Collaboration, Search for standard model Higgs bosons decaying to tau pairs, CMS Physics Analysis Summary CMS-PAS-HIG-12-043 (2012).

[20] ATLAS and CMS Collaborations, LHC Higgs Combination Group, Procedure for the LHC Higgs boson search combination in Summer 2011, Technical Report ATL-PHYS-PUB 2011-11, CMS NOTE 2011/005, (2011).

[21] CMS Collaboration, Combined results of searches for the standard model Higgs boson in pp collisions at $\sqrt{s}=7 \mathrm{TeV}$, Phys. Lett. B 710 (2012) 26, doi:10.1016/j.physletb.2012.02.064, arXiv:1202.1488.

[22] CMS Collaboration, Measurements of the properties of the new boson with a mass near $125 \mathrm{GeV}$, CMS Physics Analysis Summary CMS-PAS-HIG-13-005 (2013).

[23] CDF Collaboration, Evidence for a Narrow Near-Threshold Structure in the J/ $\psi \phi$ Mass Spectrum in $B^{ \pm} \rightarrow J / \psi \phi K^{+}$Decays, Phys. Rev. Lett. 102 (2009) 242002, 275 doi:10.1103/PhysRevLett.102.242002, arXiv:0903.2229.

[24] The CDF Collaboration, Observation of the Y(4140) structure in the J/ $\psi \phi$ Mass Spectrum in $B^{ \pm} \rightarrow J / \psi \phi K$ decays, arXiv:1101.6058.

[25] CMS Collaboration, Observation of Structures around 4148 and $4317 \mathrm{MeV}$ in the J/ $\psi \phi$ Mass Spectrum of Exclusive $B^{ \pm} \rightarrow J / \psi \phi K$ Decays, CMS Physics Analysis Summary

CMS-PAS-BPH-11-026 (2013).

[26] A. J. Buras, J. Girrbach, D. Guadagnoli, and G. Isidori, On the standard model prediction for $\mathscr{B}\left(B_{s, d}^{0} \rightarrow \mu^{+} \mu^{-}\right)$, Eur. Phys. J. C72 (2012) 2172, arXiv:1208.0934

[27] CMS Collaboration, Research for $B_{s}^{0} \rightarrow \mu^{+} \mu^{-}$and $B^{0} \rightarrow \mu^{+} \mu^{-}$decays, J. High Energy Phys. 04 (2012)033, doi:10.1007/JHEP04(2012)033, http://arxiv.org/abs/1203.3976.

[28] LHCb Collaboration, First Evidence for the Decay $B_{s}^{0} \rightarrow \mu^{+} \mu^{-}$, Phys. Rev. Lett. 110021801 (2013), doi:10.1103/PhysRevLett.110.021801, http://arxiv.org/pdf/1211.2674.

[29] CMS Collaboration, Inclusive search for supersymmetry using the razor variables in pp collisions at $\sqrt{s}=7 \mathrm{TeV}$, CMS Physics Analysis Summary CMS-PAS-SUS-11-024 (2013), arXiv:1212.6961. 
[30] CMS Collaboration, Search for supersymmetry in pp collisions at $\sqrt{s}=7 \mathrm{TeV}$ in events with a single lepton, jets, and missing transverse momentum, CMS Physics Analysis Summary

CMS-PAS-SUS-12-010 (2012), arXiv:1212.6428.

[31] CMS Collaboration, Interpretation of searches for supersymmetry with simplified models, CMS Physics Analysis Summary CMS-PAS-SUS-11-016 (2012), arXiv:1301.2175.

[32] CMS Collaboration, Search for supersymmetry in hadronic final states with missing transverse energy using the variables $\alpha_{T}$ and b-quark multiplicity in pp collisions at $\sqrt{s}=8 \mathrm{TeV}$, CMS Physics Analysis Summary CMS-PAS-SUS-12-028 (2012), arXiv:1303.2985.

[33] CMS Collaboration, Search for light stop RPV supersymmetry with three or more leptons and b-tags, CMS Physics Analysis Summary CMS-PAS-SUS-13-003 (2013).

[34] CMS Collaboration, Search for Supersymmetry in pp collisions at $8 \mathrm{TeV}$ in events with a single lepton, multiple jets and b-tags, CMS Physics Analysis Summary CMS-PAS-SUS-13-007 (2013).

[35] CMS Collaboration, Search for gluino-mediated bottom- and top-squark production in pp collisions at $8 \mathrm{TeV}$, CMS Physics Analysis Summary CMS-PAS-SUS-12-024 (2013).

[36] A. Arbey, M. Battaglia, F. Mahmoudi, D. Martinez Santos Supersymmetry confronts $B_{s} \rightarrow \mu^{+} \mu^{-}$: Present and future status CERN-PH-TH/2012-346, arXiv:1212.4887v1.

[37] CMS Collaboration, Search for Resonances in the Dilepton Mass Distribution in pp Collisions at $\sqrt{s}$ = $8 \mathrm{TeV}$, CMS Physics Analysis Summary CMS-PAS-SUS-12-061 (2013).

[38] https://twiki.cern.ch/twiki/bin/view/CMSPublic/PhysicsResultsEXO

[39] T. Matsui and H. Satz, J/ $\psi$ suppression by quark-gluon plasma formation Phys. Lett. B 178, 416 (1986).

[40] CMS Collaboration, Observation of Sequential Y Suppression in PbPb Collisions, Phys. Rev. Lett. 109222301 (2012), doi:10.1103/PhysRevLett.109.222301, http://arxiv.org/abs/1208.2826. 